\title{
Will regulatory issues continue to be a major barrier to access to bedaquiline and delamanid?
}

\author{
Tiziana Masini ${ }^{1}$, Janika Hauser ${ }^{2}$, Rutendo Kuwana ${ }^{3}$, Nguyen Nhat Linh ${ }^{4}$ and \\ Ernesto Jaramillo ${ }^{4}$
}

Affiliations: ${ }^{1}$ Independent Consultant, Italy. ${ }^{2}$ Aarhus University, Aarhus, Denmark. ${ }^{3}$ Essential Medicines and Health Products, World Health Organization, Geneva, Switzerland. ${ }^{4}$ Global TB Programme, World Health Organization, Geneva, Switzerland.

Correspondence: Tiziana Masini. E-mail: tizianamasinidgmail.com

@ERSpublications

National regulatory approval of life-saving TB drugs to be streamlined via improved communication and information sharing http://ow.ly/8Ti130i8mID

Cite this article as: Masini T, Hauser J, Kuwana R, et al. Will regulatory issues continue to be a major barrier to access to bedaquiline and delamanid? Eur Respir J 2018; 51: 1702480 [https://doi.org/10.1183/ 13993003.02480-2017].

\section{Introduction}

In 2016, 600000 people are estimated to have been eligible for multidrug-resistant (MDR) tuberculosis (TB) treatment [1]. Bedaquiline and delamanid are the first two new anti-TB compounds to have become available in over four decades and were approved by stringent regulatory authorities ${ }^{\mathrm{a}}$ in 2012 and 2014 respectively $[3,4]$. The global introduction of bedaquiline and delamanid has not kept pace with the dire need for these potentially life-saving drugs $[5,6]$. Despite a number of initiatives intending to enable access [7-14], the number of patients who have received these drugs remains unacceptably low. Very few MDR-TB high burden countries have fully integrated either bedaquiline or delamanid into their National TB Programmes (NTPs) $[1,15,16]$. While costs and concerns over adverse events may have contributed to hesitation in the adoption of bedaquiline and delamanid, a frequently cited cause for delay is the lack of national regulatory approval. ${ }^{\mathrm{b}}$ Although subject to an often long process, regulatory approval is meant to serve a public good and remains essential for sustainable import, programmatic use and therefore patient access to the drugs [17]. Despite being referenced in past reports [18], the challenges faced by drug manufacturers and National Medicines Regulatory Authorities (NMRAs) when registering the drugs have not yet been investigated more completely.

Providing an update of the current regulatory status of both bedaquiline and delamanid, we discuss recent findings from surveys and interviews conducted with NTPs and NMRAs of the 30 MDR-TB high burden

\footnotetext{
${ }^{a}$ A stringent regulatory authority is defined as a regulatory authority which is: 1) a member of the International Council for Harmonisation of Technical Requirements for Pharmaceuticals for Human Use (ICH), being the European Commission, the US Food and Drug Administration and the Ministry of Health, Labour and Welfare of Japan, also represented by the Pharmaceuticals and Medical Devices Agency (as before October 23, 2015); or 2) an ICH observer, being the European Free Trade Association, as represented by Swissmedic, and Health Canada (as before October 23, 2015); or 3) a regulatory authority associated with an ICH member through a legally binding, mutual recognition agreement, including Australia, Iceland, Liechtenstein and Norway (as before October 23, 2015) [2].

${ }^{b}$ Post-registration and implementation challenges such as affordable procurement and effective market control and pharmacovigilance programmes should be comprehensively addressed as part of a holistic access strategy.

Received: Sept 292017 | Accepted after revision: Jan 112018

Conflict of interest: None declared.

Copyright @ERS 2018
} 
TABLE 1 Registration status of bedaquiline and delamanid globally ${ }^{\#, \text { I }}$

Countries in which the medicines have been registered ${ }^{+}$

Countries in which regulatory dossiers have been filed

Bedaquiline Armenia, China, EU, ${ }^{\S}$ Hong Kong, India, Moldova, New Zealand, Peru, Philippines, Russia, South Africa, South Korea, Taiwan, Thailand, Turkey, Turkmenistan, USA, Uzbekistan

Delamanid EU, ${ }^{\S}$ Hong Kong, India, Japan, Philippines, South Korea, Turkey

Bangladesh, Brazil, Burundi, ${ }^{f}$ Cameroon, ${ }^{f}$ Ethiopia, ${ }^{f}$ Ghana, ${ }^{f}$ Indonesia, Japan, Kenya, ${ }^{f}$ Mexico, Nigeria, ${ }^{f}$ Rwanda, ${ }^{f}$ Tanzania, ${ }^{f}$ Uganda, ${ }^{f}$ Vietnam

China, Indonesia, Peru, South Africa

\#: data are up to date as of October 2017. ๆ: the countries in italics are multidrug-resistant tuberculosis high burden countries. ${ }^{+}$: in Colombia, bedaquiline is in the country's list of "Medicamentos Vitales No Disponibles", as an interim alternative for local registration; and in Macau, bedaquiline is considered to be registered in the country, via import licensing, although no formal regulatory approval was pursued by Jannsen.

$\S$ : number of countries included in European Medicines Agency approval is $31 .{ }^{f}$ : countries are part of the World Health Organization collaborative registration procedure for stringent regulatory authority-approved products.

countries and with manufacturers (October 2016 to May 2017). ${ }^{\text {c,d }}$ It should be noted that while we believe the data to be broadly representative across high burden countries, they cannot be generalised to specific context in all countries. Aside from providing an insight on hurdles and catalysts to the regulatory approval of bedaquiline and delamanid, we identify ongoing and potentially facilitative interventions to speed up in-country registration for these new drugs and future innovations.

\section{Status of registration of bedaquiline and delamanid globally and in MDR-TB high burden countries}

As of October 2017, bedaquiline had been registered in 48 countries while delamanid had been registered in 37 countries globally (table 1). It is noteworthy that no low-income countries and only a minority of middle-income countries have regulatory approvals for either drug in place. As of October 2017, dossiers for the registration of bedaquiline had been submitted by Janssen Therapeutics in another 15 countries (of them six MDR-TB high burden countries; five low- and nine middle-income countries); and dossiers for registration of delamanid had been filed in another four countries (all MDR-TB high burden middleincome countries). Licence agreements have been signed by Janssen with JSC Pharmstandard in 2013 for production and commercialisation of bedaquiline in The Commonwealth of Independent States (CIS), Georgia, Turkmenistan and Ukraine [20]. Otsuka signed licence agreements with 1) R-Pharm JSC in July 2017 to register delamanid in Russia and to expand registration and commercialisation of delamanid in other CIS countries [21]; and 2) with Mylan in August 2017 to prioritise access of delamanid in South Africa, India and 13 other countries ${ }^{\mathrm{f}}$ with high TB and/or MDR-TB burden [22]. While Mylan has filed a delamanid regulatory dossier in South Africa and successfully registered the drug in India, Pharmastandard's attempts at registering bedaquiline in Kyrgyzstan, Kazakhstan and Azerbaijan have been rejected on account of the lack of phase III clinical trial data. ${ }^{\mathrm{g}}$

\section{Manufacturer perspectives on bedaquiline and delamanid registration}

Given the limited number of dossiers to have been submitted by Janssen and Otsuka to MDR-TB high burden country NMRAs, representatives from both manufacturers were asked about key prioritisation criteria and hurdles. ${ }^{\mathrm{h}}$ The Otsuka representative described that a key priority of the company is the registration of delamanid in countries that are not eligible for drug procurement through the Global Drug

${ }^{\mathrm{c}}$ A total of 29/30 responses from NTPs and 20/30 responses from NMRAs were received during the survey period.

${ }^{\mathrm{d}}$ Our report covers the situation ahead of the release of the phase III randomised trial results from Otsuka Pharmaceuticals at the 48th UNION World Conference on Lung Health on October 13, 2017. An expedited review of these data led the World Health Organization to refine the use of delamanid under programmatic conditions. The position statement also reiterated that delamanid should be retained in the country essential medicines lists and on the list of products for medicine procurement [19].

'Includes Armenia, Azerbaijan, Belarus, Kazakhstan, Kyrgyzstan, Moldova, Russia, Tajikistan and Uzbekistan.

${ }^{\mathrm{f}}$ Afghanistan, Bangladesh, Brazil, Cambodia, DR Congo, Ethiopia, Kenya, Mozambique, Nigeria, Pakistan, Tanzania, Uganda and Zimbabwe.

${ }^{g}$ Rejection of Pharmstandard dossier for registration of bedaquiline in Azerbaijan and Kazakhstan was confirmed by NMRA Azerbaijan and NTP Kazakhstan; rejection of the same dossier in Kyrgyzstan is broadly referred to in the literature [23].

${ }^{\mathrm{h}}$ It should be noted that this analysis does not take into account the perspectives of Pharmstandard, R-Pharm and Mylan. 
Facility (i.e. a major focus on China, Russia and South Africa, which account for $26 \%$ of the global drugresistant TB burden) and in countries where clinical trials had taken place. Although dossiers have been filed in Peru and South Africa and regulatory approval was recently obtained in the Philippines, all locations of Otsuka's trial 213 [24], no dossier has been filed in Moldova, where the same trial took place. ${ }^{\text {i }}$

Janssen meanwhile places an emphasis on total case load of potentially eligible patients and on the existence of an in-country operating company that could facilitate communication between NMRAs and the manufacturer. This is viewed as a means of streamlining the registration process, which was described as lengthy and confusing in the absence of an intermediate entity.

Otsuka similarly indicated that their approach to dossier submission had been influenced by uncertainty over country-level requirements and the need to invest significant resources in each regulatory process. Acknowledging these limitations, it seems that not only greater collaboration and improved communication between all parties are necessary, but that licence agreements with local manufacturers or in-country partners should be more urgently and widely pursued both to meet NMRA requirements of incountry presence and to overcome communication hurdles.

Despite these hurdles, the initiation of all regulatory processes continues to hinge on manufacturer's willingness to file for regulatory approval. Despite a recent increase in submissions, the number of dossiers submitted remains disappointing, considering the scale of the epidemic and the number of patients who might benefit from these potentially life-saving drugs.

\section{Countries' perspective on the registration of bedaquiline and delamanid} A summary of key findings from the country survey is presented in table 2.

Most high burden countries have legal mechanisms in place to allow access to medicines prior to or during regulatory approval (e.g. import waivers). However, such pre-approval strategies are just interim stop-gap remedies benefiting a small number of patients.

While our findings suggest most countries' NMRAs are interested in facilitating the regulatory approval of bedaquiline and delamanid, the lack of phase III trial data is still viewed as a major hurdle, coupled with lack of capacity to assess the safety and efficacy of new medicines and put in place appropriate risk mitigation measures that would help with the introduction of medicines of potential significance to individual patients and broader public health benefits $[26,27]$.

It is also worth noting that, despite WHO encouraging countries to establish a dialogue with pharmaceutical manufacturers without prejudice to final registration outcomes [8], most NMRAs continue to place sole responsibility for initiating registration procedures with manufacturers, with NTPs generally playing a passive role. While international advocacy encouraging manufacturers to file for registration will continue, the uncertainty over regulatory processes referenced by both Janssen and Otsuka could be overcome by MDR-TB high burden countries showing signs of readiness to facilitate regulatory procedures.

Finally, the lack of urgency toward bedaquiline and delamanid approval noted among many NMRAs might be explained by the lack of a holistic country approach and communication between NMRAs and NTPs, preventing those unfamiliar with national MDR-TB crises from fully understanding the implications of regulatory approval of these drugs. Knowledge sharing and the communication of positive experiences from the registration and use of the two drugs also remains minimal between authorities in different countries, and should be pursued more proactively. Importantly, while many NTP respondents confirmed their desire to be involved in the drug registration process, this view was not as pervasive amongst their NMRA counterparts, suggesting a need for national exchanges over how to practically manage an efficient collaboration between the two bodies without compromising the independence of regulatory decisions by the NMRAs.

\section{(Expected) role of WHO and partners in facilitating implementation and/or} regulatory approval of new TB medicines

WHO has been playing a facilitative role to support countries with implementation and regulatory approvals [7, 28] including the addition of both drugs in the WHO Essential Medicines List in 2015 [14]. The WHO collaborative registration procedure (CRP) for pre-qualified medicines or medicines approved by national authorities that apply similar or more stringent requirements were established to reduce duplication of work and commit NMRAs to ensure efficient and streamlined review processes while

${ }^{\mathrm{i}}$ It should be noted that Moldova falls under Otsuka subsidiary Otsuka Novel Project GmbH's license agreement with Russian company R-Pharm. 
TABLE 2 Summary of key findings from surveys and interviews conducted with National TB Programmes (NTPs) and National Medicines Regulatory Authorities (NMRAs) of the 30 multidrug-resistant (MDR) tuberculosis (TB) high burden countries (October 2016 to May 2017)

Pre-approval access of new TB medicines"

National guidelines on the use of bedaquiline and delamanid available in 24 and 15 MDR-TB high burden countries, respectively

Three high burden countries have both bedaquiline and delamanid featured in their national essential medicines lists; seven countries have bedaquiline and four have delamanid only

Most countries $(92 \%, 24 / 26)$ reported having mechanisms in place for access to drugs under development and/or prior to national regulatory approval (e.g. compassionate use, expanded access programmes and the granting of import waivers)

All NTP respondents of countries where either drug was successfully registered or in the process of being registered, stated that pre-approval access use strongly contributed to greater confidence in the use of the drugs

\section{Anecdotal}

The NTP respondent from one country stated that the current mechanism used to access bedaquiline through import waivers for patients covered by a partner's project was no longer sufficient to cover country needs (in this country, early implementation experiences and expansion of eligibility criteria have contributed to clinician's growing willingness to prescribe bedaquiline-based regimens)

The NTP respondent from one country referenced its overall nascent drug registration procedures but expressed the country's willingness to discuss "the pros and cons of registration" if a dossier were to be submitted

The NTP respondent from one country expressed the need to continue gathering more information from in-country studies prior to the NMRA reviewing any regulatory dossier

\section{Countries' perspective on the registration of bedaquiline and delamanid}

All NMRAs were aware of both products for the treatment of MDR-TB, and there is widespread agreement over the need for regulatory approval to ensure sustainable access

Most NTP and NMRA respondents rated communication between the two bodies as neutral (67\% (12/18) and 56\% (10/18))

$40 \%$ (10/25) of countries reported some NTP involvement in the registration process

Majority of NTP respondents (73\% (19/26)] felt that their increased support in promoting the approval for these medicines would likely lead to streamlined regulatory approval; only $30 \%(6 / 20)$ of the NMRA respondents see a potential role of NTPs in streamlining the national regulatory approval

Most NMRA respondents (65\% (13/20)) stated that sole responsibility for initiating regulatory approval processes lies with manufacturers only and NMRAs are not willing to take any action prior to the submission of a manufacturer dossier

Majority of NMRA respondents (89\% (17/19)] confirmed the presence of legal frameworks for accelerated review of products; four countries indicated to have applied such mechanisms for bedaquiline registration

$33 \%(10 / 30)$ countries are participating in the WHO collaborative registration procedure; ${ }^{+}$among them, three African countries reported being part of, observing or having applied to join other regulatory harmonisation/collaborative programmes

Among the countries that reported to not participate in the WHO collaborative registration procedure or any regulatory harmonisation/ collaborative programme, 45\% (9/20) of NMRAs expressed their interest in exploring participation

High cost, pharmacovigilance and importation challenges listed as long-term challenges, even post-registration

\#: a total of 29 responses from NTPs and 20 responses from NMRAs were received during the survey period. If a respondent did not answer a question or inconsistencies with data from desk review were noted, the denominator was adjusted and the data were not taken into account in the analysis. ": data from respondents of our survey were cross-checked with Médecins Sans Frontières and Stop TB Partnership Out of Step report 2017 [15] and other data obtained from extensive desk review. ${ }^{+}$: data from respondents of our survey were cross-checked and complemented with the list of participating countries available on the World Health Organization (WHO) website [25].

guaranteeing that countries can carry out independent reviews according to their national legislation [25, 29]. Considerable enthusiasm expressed for the WHO CRP by non-participating NMRAs should thus be converted into action as soon as possible, and governments and non-governmental stakeholders are encouraged to make use of the resources and capacity building schemes on offer. WHO organises regular workshops, webinars, or has some specific spots at key conferences to promote the uptake of the CRP by marketing authorisation holders and regulators.

Despite initial challenges, the pilot CRP for bedaquiline (Sirturo $100 \mathrm{mg}$ ) is now performing well, with nine countries from the African region currently being involved (including two MDR-TB high burden countries) (table 1) and particular success in skills-sharing and consensus building (C.G. Mercè; oral presentation at the DIA 2017 Global Annual Meeting, June 2017, Chicago, IL, USA; www.diaglobal.org/ flagship/DIA-2017/Program/sched?).

Country-level capacity building needs are great. As countries move to register bedaquiline and delamanid, both NTPs and NMRAs expressed the need for further support from WHO and other stakeholders in many areas, as summarised in box 1. Joint efforts between national bodies, civil societies and WHO are 
BOX 1 Major support actions requested by National TB Programmes and/or National Medicines Regulatory Agencies (NMRAs) to World Health Organization (WHO) and stakeholders for a faster implementation of the new tuberculosis drugs

High-level advocacy to encourage manufacturers to file for regulatory approval in their country Informing and sensitising NMRAs on the importance of facilitating these approvals

Capacity building and specific training on bedaquiline and delamanid to facilitate more efficient dossier review Data sharing on implementation and regulatory approvals, as well as updates on safety and efficacy profiles WHO prequalification of bedaquiline and delamanid to both ease import and facilitate regulatory approval

necessary in order to facilitate regulatory approvals for bedaquiline, delamanid and any future innovations in the TB space.

\section{Conclusions}

Despite sometimes being a complex, lengthy process, national regulatory approval is meant to serve a public good and furthermore is often the only tool to guarantee widespread and sustainable long-term access to medicines. Despite several opportunities for fast-track registration of new anti-TB drugs, this process is complicated by lack of or ineffective communication between all parties involved. Such findings are particularly critical in the context of the global MDR-TB crisis and the limited treatment options of a growing number of patients. NTPs and other TB stakeholders can contribute to addressing these challenges by promoting harmonisation and the use of collaborative registration platforms, proactively engaging in sharing of WHO global policies on these drugs, and of the growing experience accumulated in countries making progress in their use. Externally facilitated regulatory platforms such as the WHO CRP and initiatives to promote regulatory harmonisation are not the only option in this regard. NTPs, as mandated national authorities on managing MDR-TB, are encouraged to act as mediators, particularly in updating and sensitising drug regulators about the importance of the drugs' rapid registration and postregistration oversight. While post-registration and implementation challenges such as affordable procurement and effective pharmacovigilance programmes should not be minimised but comprehensively addressed, the rapid registration of bedaquiline and delamanid is an essential first step that should be promptly initiated by the manufacturers, and promoted and pursued with urgency by all partners working to realise the targets of the End TB Strategy. In fact, responsibility lies not only with government and civil society, but also with the manufacturers whose regulatory arrangements should be more proactive to ensure that their products reach people suffering with MDR-TB. Importantly, such findings are of relevance not only for bedaquiline and delamanid, but they also provide vital insights for future innovations in the TB space and that are useful for regulatory authorities, manufacturers and practitioners working across global health issues.

\section{References}

1 World Health Organization (WHO). Global Tuberculosis Report 2017. (WHO/HTM/TB/2017.23). Geneva, WHO, 2017. www.who.int/tb/publications/global_report/en/ Last updated: October, 2017. Date last accessed: January 10, 2018.

2 World Health Organization (WHO). Proposal for updating the definition of "stringent regulatory authority". Geneva, WHO, 2017. http://www.who.int/medicines/areas/quality_safety/quality_assurance/SRA_QAS17-728Rev1_ 31082017.pdf Last updated: August, 2017. Date last accessed: January 10, 2018.

3 FDA News Release. https://wayback.archive-it.org/7993/20170112023916/http://www.fda.gov/NewsEvents/Newsroom/ PressAnnouncements/ucm333695.htm Date last updated: December 31, 2012. Date last accessed: January 10, 2018.

4 European Medicines Agency. Deltyba. www.ema.europa.eu/ema/index.jsp?curl=pages/medicines/human/medicines/ 002552/smops/Positive/human_smop_000572.jsp\&mid=WC0b01ac058001d127 Date last updated: November 21, 2013. Date last accessed: January 10, 2018.

5 Borisov SE, Dheda K, Enwerem M, et al. Effectiveness and safety of bedaquiline-containing regimens in the treatment of MDR- and XDR-TB: a multicentre study. Eur Respir J 2017; 49: 1700387.

6 Pontali E, Sotgiu G, D'Ambrosio L, et al. Bedaquiline and multidrug-resistant tuberculosis: a systematic and critical analysis of the evidence. Eur Respir J 2016; 47: 394-402.

7 World Health Organization (WHO). Policy Implementation Package for New TB Drug Introduction 2014. (WHO/HTM/TB/2014.22). Geneva, WHO, 2014. www.who.int/tb/PIPnewTBdrugs.pdf Last updated: 2014. Date last accessed: January 10, 2018.

8 World Health Organization (WHO). Companion handbook to the WHO guidelines for the programmatic management of drug-resistant tuberculosis 2014. (WHO/HTM/TB/2014.11). Geneva, WHO, 2014. http://apps. who.int/iris/bitstream/10665/130918/1/9789241548809_eng.pdf?ua=1\&ua=1 Last updated: 2014. Date last accessed: January 10, 2018.

9 The Global Fund. Focus on drug-resistant TB. www.theglobalfund.org/media/6651/publication_drug-resistant tuberculosis_focuson_en.pdf Last updated: July, 2017. Date last accessed: January 10, 2018.

10 USAID Press Office. USAID and Johnson \& Johnson to tackle antibiotic-resistant tuberculosis. https://2012-2017. usaid.gov/news-information/press-releases/dec-11-2014-usaid-and-johnson-johnson-tackle-antibiotic-resistanttuberculosis Date last updated: December 22, 2014. Date last accessed: January 10, 2018. 
11 Pontali E, D'Ambrosio L, Centis R, et al. Multidrug-resistant tuberculosis and beyond: an updated analysis of the current evidence on bedaquiline. Eur Respir J 2017; 49: 1700146.

12 Hafkin J, Hittel N, Martin A, et al. Early outcomes in MDR-TB and XDR-TB patients treated with delamanid under compassionate use. Eur Respir J 2017; 50: 1700311.

13 De Marez T. Oral presentation at the Critical Path to TB Drug Regimens Workshop 2017. www.cptrinitiative.org/ wp-content/uploads/2017/05/21_03_DeMarez.pdf Date last updated: March 21, 2017. Date last accessed: January $10,2018$.

14 World Health Organization (WHO). WHO Model List of Essential Medicines. 20th List. www.who.int/medicines/ publications/essentialmedicines/20th_EML2017_FINAL_amendedAug2017.pdf?ua=1 Last updated: August, 2017. Date last accessed: January 10, 2018.

15 Médecins Sans Frontières and Stop TB Partnership. Out of Step 2017. TB Policies in 29 Countries. A survey of prevention, testing and treatment policies and practices. www.msfaccess.org/sites/default/files/MSF_assets/TB/Docs/ TB_Report_OutOfStep 3rdEd_ENG 2017.pdf Date last updated: July 5, 2017. Date last accessed: January 10, 2018.

16 Guglielmetti L. Bedaquiline for the treatment of multidrug-resistant tuberculosis: another missed opportunity? Eur Respir J 2017; 49: 1700738.

17 Stop TB Partnership and Médecins Sans Frontières (MSF). Country perceptions for implementation of the new multi-drug resistant TB (MDR-TB) medicine. Report. A survey conducted among the 27 high MDR-TB burden countries. March-July 2015. www.stoptb.org/assets/documents/resources/publications/technical/Report\%20of\% 20the\%20survey\%20on\%20new\%20MDR-TB\%20drugs\%20final\%2009032016.pdf Last updated: 2016. Date last accessed: January 10, 2018.

18 Wileman H, Mishra A. Drug lag and key regulatory barriers in the emerging markets. Perspect Clin Res 2010; 1: 51-56.

19 World Health Organization (WHO) 2018 WHO position statement on the use of delamanid for multi-drug resistant tuberculosis. www.who.int/tb/publications/2018/WHOPositionStatementDelamanidUse.pdf?ua=1 Last updated: January 2018. Date last accessed: January 17, 2018.

20 Janssen Pharmaceutica NV. Janssen announces agreements to expand research and development efforts in Russia. www.janssen-emea.com/sites/default/files/PressReleases/Janssen\%20Announces\%20Agreements\%20to\%20Expand\% 20Research\%20and\%20Development\%20Efforts\%20in\%20Russia.pdf Date last updated: January 18, 2013. Date last accessed: January 10, 2018.

21 Otsuka. Press Release. Otsuka and R-Pharm announce licensing agreement to commercialize deltyba ${ }^{\mathrm{TM}}$ (Delamanid) for multidrug-resistant tuberculosis (MDR-TB) in Russia and CIS countries. www.otsuka.co.jp/en/company/ newsreleases/assets/pdf/20170718_1.pdf Date last updated: July 18, 2017. Date last accessed: January 10, 2018.

22 Mylan. Press release. Otsuka and Mylan announce license agreement to commercialize delamanid (Deltyba TM) for multidrug-resistant tuberculosis (MDR-TB) in high-burden countries. http://newsroom.mylan.com/ 2017-08-24-Otsuka-and-Mylan-Announce-License-Agreement-to-Commercialize-Delamanid-Deltyba-TM-for-MultidrugResistant-Tuberculosis-MDR-TB-in-High-Burden-Countries Date last updated: August 24, 2017. Date last accessed: January 10, 2018.

23 Médecins Sans Frontières (MSF). DR-TB Drugs Under the Microscope, 4th edition. Geneva, MSF, 2016. www. msfaccess.org/content/report-dr-tb-drugs-under-microscope-4th-edition Last updated: July 2016. Date last accessed: January 10, 2018.

24 ClinicalTrials.gov. A service of the U.S. National Institutes of Health. https://clinicaltrials.gov/ct2/show/ NCT01424670. Date last accessed: January 10, 2018.

25 WHO Collaborative Procedure for Accelerated Registration. https://extranet.who.int/prequal/content/collaborativeprocedure-accelerated-registration Date last accessed: January 10, 2018.

26 World Health Organization (WHO). The use of delamanid in the treatment of multidrug-resistant tuberculosis in children and adolescents: interim policy guidance. (WHO/HTM/TB/2016.14). Geneva, WHO, 2016. http://apps.who int/iris/bitstream/10665/250614/1/9789241549899-eng.pdf Last updated: 2016. Date last accessed: January 10, 2018.

27 World Health Organization (WHO). The use of bedaquiline in the treatment of multidrug-resistant tuberculosis Interim policy guidance. (WHO/HTM/TB/2013.6). Geneva, WHO, 2013. http://apps.who.int/iris/bitstream/10665/ 84879/1/9789241505482_eng.pdf?ua=1 Last updated: 2013. Date last accessed: January 10, 2018.

28 World Health Organization (WHO). Active tuberculosis drug-safety monitoring and management (aDSM) Framework for implementation. (WHO/HTM/TB/2015.28). Geneva, WHO, 2015. http://apps.who.int/iris/ bitstream/10665/204465/1/WHO_HTM_TB_2015.28_eng.pdf Last updated: 2015. Date last accessed: January 3, 2017.

29 Mercè CG. Accelerating regulatory approvals through the World Health Organization collaborative registration procedures. Pharmaceuticals Policy and Law 2016; 18: 109-120. 